\title{
HPV DNA genotyping test in low and high grade intraephitelial lesions
}

\author{
Maria Gabriella Lepore, Vittorio Focarelli,Vincenzo Rondinelli, Stefania Giglio, Sandra Castagna, Paolo Opipari, \\ Raffaele Saraceno, Pasqualina Chiefalo, Simona Roccia, Rosanna Masciari \\ Virologia e Microbiologia \\ Azienda Ospedaliera Pugliese-Ciaccio, Presidio Pugliese,Via Pio X, 88100 Catanzaro
}

Key words: HPV DNA test, cervical pathology, high risk, low risk

Genotipizzazione di HPV DNA nelle lesioni intraepiteliali di basso e alto grado

\section{SUMMARY}

The human papillomavirus (HPV) is certainly one of the few viruses associated with cancer and is present in a significant number of cancers of the anus, penis, vagina, vulva and in almost all cervical cancers. The aim of our work is to evaluate the impact of genotypes high-risk oncogenic (HR) in low-grade (LSIL) and high grade (HSIL) intraepithelial lesions. In it are also evaluated the impact of genotypes low oncogenic risk (LR) and the percentage of non-detection (NR) Papillomavirus DNA

Between January 2008 and June 2009 we submitted for HPV DNA genotyping test 392 patients, seronegative for HIVI/2, of which 278 with LSIL and II 4 with HSIL.

In 250 patients tested positive for HPV DNA HR the type 16 is present in 102 patients (4I\%), the 18 in 62 (25\%), the 31 in 37 patients (I5\%), the 33 in 13 patients (5\%); also was limited the simultaneous presence of 16 and 18 ( 13 patients $5 \%$ )

The search for HPV DNA is a modern method of study of cervical pathology. Optimizes the follow-up of intraepithelial lesions and the therapeutic intervention, avoiding the dangers of over/ undertreatment and allows to monitor women treated for cervical pathology.

Il Papillomavirus umano (HPV) (Figura I) appartiene alla famiglia dei Papovaviridae, genere Papillomavirus; il genoma è costituito da DNA bicatenario di circa 8000 pb, avvolto da un capside di natura proteica.

È uno dei pochi virus, insieme a quelli dell'epatite B (HBV) e dell'epatite C (HCV), al virus di Epstein Barr (EBV), ai retrovirus HTLV I/II ed all'Herpes virus di tipo 8 (HHV8) certamente associati a tumori ed è presente in un numero considerevole di carcinomi di ano, pene, vagina, vulva e nella quasi totalità dei carcinomi del collo dell'utero.

HPV costituisce infatti un fattore necessario, anche se non sufficiente, per lo sviluppo del carcinoma cervicale che è, dopo il carcinoma mammario, il secondo cancro a più alta incidenza nella popolazione femminile.

Il processo di oncogenesi è innescato dalle oncoproteine virali E6 ed E7 che si legano rispettivamente agli oncosoppressori cellulari p53 ed $\mathrm{Rb}$ degradandoli, inducendo in tal modo proliferazione e immortalizzazione delle cellule umane.

Scopo del nostro lavoro è quello di valutare l'incidenza dei virotipi ad alto rischio oncogeno (H.R.) nelle lesioni intraepiteliali di basso grado (LSIL) e di alto grado (HSIL). In esso vengono anche valutate l'incidenza dei virotipi a basso rischio oncogeno (L.R.) e la percentuale di non rilevazione (N.R.) del DNA del Papillomavirus

Nel periodo gennaio 2008-giugno 2009 abbiamo sottoposto alla genotipizzazione dell'HPV DNA 392 pazienti, sieronegative per HIV1/2, di cui 278 (età media 33 anni) con LSIL e 114 (età media 47 anni) con HSIL. I campioni sono stati raccolti con la tecnica del brushing cervicale, conservati nei flaconi di ThinPrep (2) per citologia liquida. Essi sono in tal modo stabili 21 giorni a temperatura ambiente o possono essere conservati a $2-8^{\circ} \mathrm{C}$ per 12 settimane. Il kit utilizzato è Linear Array HPV Genotyping Test della Roche che discrimina 37 genotipi virali ad alto e basso rischio (Figura II). In 150 delle 278 pazienti (54\%) con LSIL sono presenti genotipi ad alto rischio oncogeno (H.R.); in 91 (33\%) quelli a basso rischio (L.R.) ed in $37(13 \%)$ non viene rilevato il DNA di HPV.

In ben 100 delle 114 pazienti (88\%) con HSIL sono presenti genotipi ad alto rischio oncogeno (H.R.); solo in 8 (7\%) quelli a basso rischio (L.R.) ed in $6(5 \%)$ non viene rilevato il DNA di HPV.

Nelle 250 pazienti risultate positive per HPV DNA H.R. il virotipo 16, identificato nel 1995 come virus oncogeno dalla International Agency for Reaserch on Cancer, è presente in 102 pazienti $(41 \%)$, il 18 in $62(25 \%)$, il 31 in 37 pazienti (15\%); molto contenuta la presenza del 33 (13 pazienti 5\%) così come è risultata limitata la contemporanea presenza del 16 e 18 (13 pazienti 5\%).

Le percentuali di positività dei genotipi ad alto rischio sono perfettamente confrontabili con quelle riportate dalla letteratura più recente (3).

La ricerca del DNA di HPV costituisce un moderno metodo di studio della patologia cervicale.

Il Pap-test, da solo, è oggi superato: la sua sensibilità è pari all' $80 \%$ il che significa che il $20 \%$ delle lesioni, specie se topograficamente limitate, sfugge alla diagnosi.

Pap-test ed HPV DNA (4), eseguiti contemporaneamente, raggiungono invece una sensibilità quasi del $100 \%$ perché si riduce il tasso di falsi negativi. Essi consentono inoltre l'allungamento del tempo di intervallo dello screening ed hanno un alto valore predittivo negativo (donne negative ad entrambi i test hanno un rischio di sviluppare il tumore inferiore allo $0,2 \%$ ).

HPV test ha un basso valore predittivo positivo nelle giovani donne in cui è alta la prevalenza dell'infezione ma anche la possibilità di una sua eradicazione spontanea; ha, in caso di risultato persistentemente positivo, un alto valore predittivo di lesione ed il rischio di evoluzione neoplastica a due anni è circa 200 volte maggiore rispetto alle donne negative.

Il test di genotipizzazione consente di rilevare e monitorare le infezioni multiple sostenute da più genotipi, di discriminare tra infezioni sostenute da genotipi ad alto e basso rischio e di monitorare la persistenza di infezione da genotipi ad alto rischio, persistenza che notoriamente rappresenta il fattore di rischio più importante per il carcinoma cervicale.

Permette ancora di ottimizzare il follow-up delle lesioni intraepiteliali per predirne la regressione, persistenza o progressione; di calibrare (1) l'intervento terapeutico evitando pericoli di over/undertreatment e di monitorare le pazienti sottoposte a laserterapia o a conizzazione.

\section{Corresponding author: Rosanna Masciari}

Via Luigi Pascali, 30 88100 Catanzaro

Tel. 0961/725069 - Fax 0961/883390

E-mail: r.masciari@tele2.it 


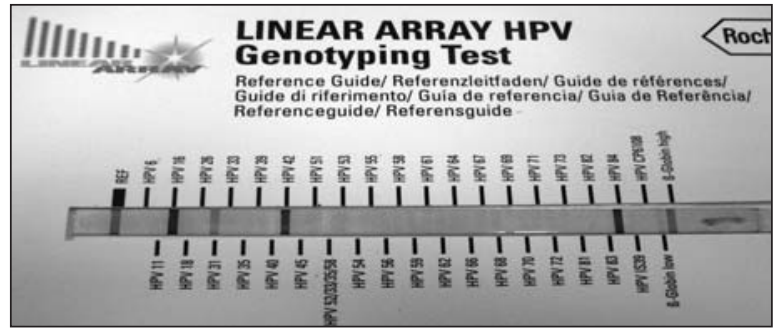

Figura I. Test positivo per 4 genotipi (2 H.R. e 2 L.R.)

\section{BIBLIOGRAFIA}

1. Cuzick J., Szarewski A., Cubie H. et al.: Management of women who test positive for high-risk types of human papilloma virus: the HART study. Lancet, 362: 1871-6, 2003;

2. Schledermann D., Andersen B.T., Bisgard K. et al.: Are adjunctive useful in routine cervical cancer screening. Application of p16 and HPV-PCR on Thin Prep samples with histological follow-up. Diagnostic Cytopathology, 2008;

3. Smith J.S., Lindsay L., Hoots B. et al.: Human papillomavirus type distribution in invasive cervical cancer and high-grade cervical lesions. A metaanalysis update. Int. J. Cancer, 121: 621-32, 2007;

4. Wright T.C. et al.: Adding a test for human papilloma virus DNA to cervical-cancer screening. N. Engl. J. Med., Feb. 6; 348 (6), 489-90, 2003 